\title{
Long-term lifestyle intervention lowers the incidence of stroke in Japanese patients with type 2 diabetes: a nationwide multicentre randomised controlled trial (the Japan Diabetes Complications Study)
}

\author{
H. Sone $\cdot$ S. Tanaka $\cdot$ S. Iimuro $\cdot$ S. Tanaka $\cdot$ K. Oida $\cdot$ \\ Y. Yamasaki $\cdot$ S. Oikawa $\cdot$ S. Ishibashi $\cdot$ S. Katayama $\cdot$ \\ H. Yamashita $\cdot$ H. Ito $\cdot$ Y. Yoshimura $\cdot$ Y. Ohashi $\cdot$ Y. Akanuma \\ N. Yamada for the Japan Diabetes Complications Study Group
}

Received: 12 July 2009 / Accepted: 21 October 2009 /Published online: 7 January 2010

(C) The Author(s) 2009. This article is published with open access at Springerlink.com

\begin{abstract}
Aims/hypothesis The aim of the study was to clarify whether a therapeutic intervention focused on lifestyle modification affected the incidence of vascular complications in patients with established diabetes.

Methods A total of 2,033 eligible Japanese men and women aged 40-70 years with type 2 diabetes from 59 institutes were randomised to a conventional treatment group (CON), which continued to receive the usual care, and a lifestyle intervention group (INT), which received
\end{abstract}

Electronic supplementary material The online version of this article (doi:10.1007/s00125-009-1622-2) contains a list of members of the Japan Diabetes Complications Study Group, which is available to authorized users.

H. Sone $\cdot$ N. Yamada $(\bowtie)$

Department of Internal Medicine,

University of Tsukuba Institute of Clinical Medicine,

1-1-1 Tennodai,

Tsukuba, Ibaraki, Japan 305-8575

e-mail: jdcstudy@md.tsukuba.ac.jp

S. Tanaka

Laboratory of Biostatistics, Tokyo University of Science,

Tokyo, Japan

S. Iimuro $\cdot$ Y. Ohashi

Department of Biostatistics, University of Tokyo School of Medicine,

Tokyo, Japan

S. Tanaka

Translational Research Center, Kyoto University,

Kyoto, Japan

K. Oida

Fukui Chuo Clinic,

Fukui, Japan education on lifestyle modification regarding dietary habits, physical activities and adherence to treatment by telephone counselling and at each outpatient clinic visit, in addition to the usual care. Randomisation and open-label allocation were done by a central computer system. Primary analysis regarding measurements of control status and occurrence of macro- and microvascular complications was based on 1,304 participants followed for an 8 year period.

Results Although status of control of most classic cardiovascular risk factors, including body weight, glycaemia, serum lipids and BP, did not differ between groups during

\footnotetext{
Y. Yamasaki

Center for Advanced Science and Innovation, Osaka University, Osaka, Japan

S. Oikawa

Department of Medicine, Nippon Medical School,

Tokyo, Japan

S. Ishibashi

Department of Endocrinology and Metabolism,

Jichi Medical College,

Tochigi, Japan

S. Katayama

The Fourth Department of Medicine, Saitama Medical School,

Saitama, Japan

H. Yamashita

Department of Ophthalmology,

Yamagata University School of Medicine,

Yamagata, Japan
} 
the study period, the incidence of stroke in the INT group (5.48/1,000 patient-years) was significantly lower than in the CON group $(9.52 / 1,000$ patient-years) by KaplanMeier analysis ( $p=0.02$ by logrank test) and by multivariate Cox analysis (HR 0.62, 95\% CI 0.39-0.98, $p=0.04$ ). The incidence of CHD, retinopathy and nephropathy did not differ significantly between groups. Lipoprotein(a) was another significant independent risk factor for stroke.

Conclusions/interpretation These findings suggest that lifestyle modification had limited effects on most typical control variables, but did have a significant effect on stroke incidence in patients with established type 2 diabetes.

\section{Clinical Trial Registration: UMIN-CTR C000000222}

Funding: The Ministry of Health, Labour and Welfare, Japan

Keywords Complications · Lifestyle intervention · Patient education $\cdot$ Stroke

\section{Abbreviations \\ CON Conventional treatment group \\ CVD Cardiovascular disease \\ IGT Impaired glucose tolerance \\ INT Lifestyle intervention group \\ JDCS The Japan Diabetes Complications Study}

\section{Introduction}

Lifestyle management through patient education plays a crucial role in prevention and care of diabetes. It is well established that lifestyle interventions, including diet and/or exercise, can prevent type 2 diabetes [1-6] as well as ameliorate glycaemia and other risk factors for complications [7-12] in patients with established diabetes. Recent reports of two studies that examined the effect of a lifestyle intervention on individuals with impaired glucose tolerance (IGT) over a long-term follow-up period (the China Da Qing Diabetes Prevention Study [13] and the Finnish Diabetes Prevention Study [14]), failed to show significant effects on cardiovascular disease (CVD) events or mortality. However, it is not known whether an intervention

\section{H. Ito}

Tokyo Metropolitan Geriatric Hospital,

Tokyo, Japan

\section{Y. Yoshimura}

Department of Nutrition, Shikoku University,

Tokushima, Japan

Y. Akanuma

The Institute for Adult Diseases, Asahi Life Foundation,

Tokyo, Japan mainly focusing on lifestyle modification through patient education could prevent the occurrence of complications in patients with established diabetes, although there have been a few studies $[15,16]$ on lifestyle modification in combination with pharmacotherapy for hyperglycaemia, hypertension and dyslipidaemia in patients with type 2 diabetes.

The Japan Diabetes Complications Study (JDCS), a nationwide randomised controlled study of Japanese patients with type 2 diabetes, was designed to clarify whether a long-term therapeutic intervention mainly focused on lifestyle education has an effect on the incidence of diabetic macro- and microvascular complication events in patients with established type 2 diabetes (see Electronic supplementary material for members of JDCS). Another aim of this study was to clarify pathophysiological characteristics in East Asian patients with type 2 diabetes [17-20]. We previously published a 3 year interim report [21] showing significant but only limited improvement in glycaemia and no improvement in body weight, BP and serum lipids as a result of lifestyle modifications in patients with type 2 diabetes. This result was quite consistent with other subsequent studies with similar observation periods $[8,11$, $22,23]$. The present report shows results after 8 years of an investigation that focused on the incidence of macro- and microvascular complications of diabetes.

\section{Methods}

Recruitment of patients Participants in the study were previously diagnosed patients with type 2 diabetes aged 40-70 years whose $\mathrm{HbA}_{1 \mathrm{c}}$ levels were $\geq 6.5 \%$. From outpatient clinics in 59 university and general hospitals nationwide that specialise in diabetes care, a total of 2,205 patients (mean age 58.6 years; $47 \%$ women) were initially registered from January 1995 to March 1996. Excluded were patients with a history of angina pectoris, myocardial infarction, stroke, peripheral arterial disease, familial hypercholesterolaemia, type III hyperlipidaemia, non-diabetic nephropathy, nephrotic syndrome, pre-proliferative and proliferative retinopathy, intra-ocular surgeries, serum creatinine levels $>120 \mu \mathrm{mol} / \mathrm{l}$, and mean values of two spot urine examinations for an albumin excretion rate of $<150 \mathrm{mg} / \mathrm{g}$ creatinine. Diabetes mellitus and IGT were diagnosed according to the Report of the Committee of the Japan Diabetes Society on the Classification and Diagnostic Criteria of Diabetes Mellitus, which is almost identical in terms of cut-off values for glucose levels to those of the WHO. The protocol for the study, which is in accordance with the Declaration of Helsinki and the Ethical Guidelines for Clinical/Epidemiological Studies of the Japanese Ministry of Health, Labour and Welfare, received ethical 
approval from the institutional review boards of all of the participating institutes (RCT registration number was C000000222 in www.umin.ac.jp). Written informed consent was obtained from all patients enrolled.

Allocation of patients Before April 1996, when the intervention began, patients who did not meet the eligibility criteria were excluded. Finally, a total of 2,033 patients aged $58.5 \pm 6.9$ years and who had diabetes for a duration of $10.9 \pm 7.2$ years (both mean $\pm \mathrm{SD}$ ) were included from the present analysis. Figure 1 is a flow diagram of the JDCS. Patients were allocated randomly into either a lifestyle intervention (INT) group or a conventional treatment $(\mathrm{CON})$ group. Randomisation and all analyses were done by a central computer at our database centre. This study was open-labelled and the interventions for the INT group were continued until March 2003.

Lifestyle intervention As basal therapeutic management of all patients in both the CON and INT groups, regular specialists' care was provided throughout the study period and patients were treated as they were before the study started. This included dietary advice by an administrative dietitian, using the 'Food Exchange Lists Dietary Guidance for Persons with Diabetes' [24].

In addition to this routine conventional treatment, education of patients in the INT group was given through individual counselling on dietary habits, physical activities and adherence to treatment, including taking medicine properly. Counselling was provided by physicians, nurses, dietitians and other co-medical staff during each outpatient clinic visit. Patients in the INT group had a typically 5-10 min longer interview than the patients in the CON group at each clinic visit for a discussion on possible causes of any changes in $\mathrm{HbA}_{1 \mathrm{c}}$ levels, weight and other control variables from the previous visit, with emphasis on lifestyle changes. For example, when it was revealed that control of glycaemic and other variables had worsened, that dietary intake, including quantity and content, and alcohol intake had changed, that patterns of physical activity had changed or that patients tended to forget to take their medicine, possible strategies for improving lifestyle and habits were discussed. Furthermore, patients in the INT group also received additional advice regarding one or two particular topic(s) at each visit and were given educational material consisting of 23 brochures that discussed various aspects of diabetes care with an emphasis on the importance of lifestyle and behavioural changes such as 'Why am I obese even if I do not eat so much?', 'Tips for continuing exercise', 'What kinds of stress affect the control of diabetes' or 'Is your triglyceride level OK?'.

Patients in the INT group also received 15 min telephone counselling sessions at least once every 2 weeks by nurses, dietitians and psychotherapists who were trained in diabetes education. These telephone sessions were performed based on a structured and uniform format. Additional counselling sessions were encouraged at any convenient time, depending on the needs of patients in the INT group. A diary to record the progress of laboratory and other data was distributed to the INT patients to provide better feedback on therapeutic results. A pedometer was also distributed to INT patients for objective exercise assessment.

Goals were set for patients in the INT group and their physicians: i.e. $\mathrm{HbA}_{1 \mathrm{c}}$ level $<6.5 \%$; $\mathrm{BMI}<22 \mathrm{~kg} / \mathrm{m}^{2}$; BP $<140 / 85 \mathrm{mmHg}$; serum cholesterol level $<5.72 \mathrm{mmol} / \mathrm{l}$; serum triacylglycerol level $<1.65 \mathrm{mmol} / \mathrm{l}$; serum HDLcholesterol $>1.04 \mathrm{mmol} / \mathrm{l}$; WHR $<0.9$ for men and $<0.8$ for women; smoking cessation; and abstinence from alcohol. Goals regarding BP and serum cholesterol levels were updated in accord with the revision of guidelines made by the Japan Diabetes Society, which were $<130 / 80 \mathrm{mmHg}$ and $<5.17 \mathrm{mmol} / 1$, respectively.

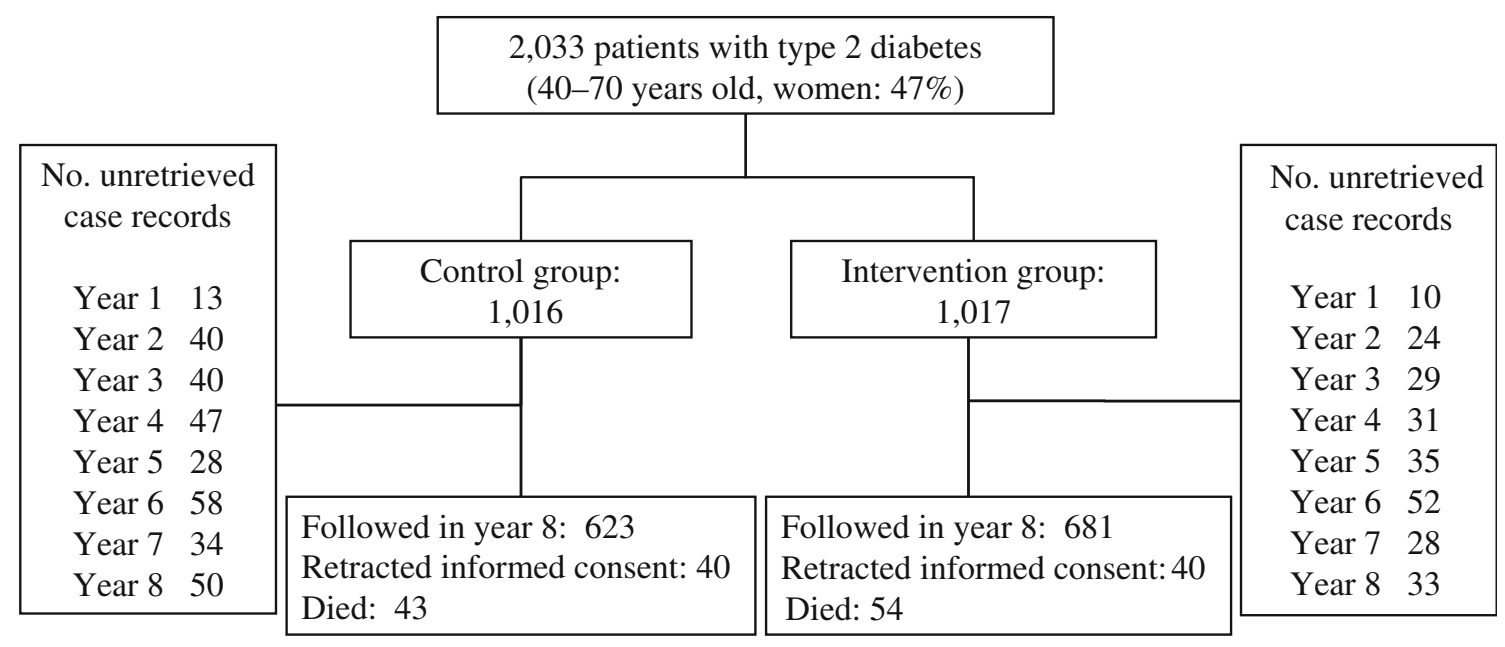

Fig. 1 Flow diagram of the JDCS 
During the study period, patients in the INT group with poor control $\left(\mathrm{HbA}_{1 \mathrm{c}}>8.0 \%\right.$, serum total cholesterol level $>5.72 \mathrm{mmol} / \mathrm{l}$, serum triacylglycerol level $>1.65 \mathrm{mmol} / 1$ or BMI $>22 \mathrm{~kg} / \mathrm{m}^{2}$ ) were identified and sent additional educational material. At the same time, their physicians were encouraged to strengthen the intervention through increasing time for education by telephone or at clinic visits or recommending hospital admissions for education. Changes in medication including insulin and oral antihypertension/dyslipidaemia agents were not restricted in either group and were made based on therapeutic necessity.

Assessment of lifestyle Extensive lifestyle surveys were performed at baseline and 5 years after the start of the intervention in both groups. We used detailed questionnaires for patients to determine dietary (including alcohol drinking) content, amount of exercise and smoking rate. The dietary survey comprised food records and a food frequency questionnaire, results of which were analysed by an administrative dietitian using standardised software for population-based surveys and nutrition counselling in Japan (EIYO-KUN v.4.5, manufactured at Shikoku University Nutrition Database) [25] based on the Standard Tables of Food Composition in Japan [26] edited by the Japanese Ministry of Education, Culture, Sports, Science and Technology. Physical activity was determined by a questionnaire that inquired about types of exercise (walking, jogging, tennis, etc) and average time (min) spent exercising per day at baseline and the Baecke's Total Physical Activity Index [27] at the fifth year.

Clinical and laboratory measurements Mean values of at least two measurements each year were obtained for the following variables: $\mathrm{HbA}_{1 \mathrm{c}}$, fasting plasma glucose/insulin/ C-peptide, serum lipids/creatinine/urea nitrogen, and urine analysis. All other measurements including those for body weight, BP and WHR and a neurological/ophthalmological examination were done at least once yearly, with a mean value obtained if measurements were done twice a year. $\mathrm{HbA}_{1 \mathrm{c}}$ assays were standardised by the Laboratory Test Committee of the Japan Diabetes Society, with 5.8\% as the upper normal limit. All other laboratory tests were done by standard methods in each clinic. Electrocardiograms and chest $\mathrm{x}$-rays were performed annually. Patients were assessed yearly after the baseline evaluation.

Endpoints of the study Primary outcomes of the study were development and progression of microangiopathy and occurrence of macrovascular complication events. Microangiopathy endpoints consisted of those related to retinopathies and nephropathies. Retinopathy was evaluated by qualified ophthalmologists using the following classification designed for this research: Stage 0, no retinopathy;
Stage 1, haemorrhage and hard exudates; Stage 2, soft exudates; Stage 3, intraretinal microvascular abnormalities and venous changes including beading, loop and duplication; Stage 4 , new vessels, vitreous haemorrhage, fibrous proliferation and retinal detachment. The retinopathy endpoint was (1) development of retinopathy (from Stage 0 to any other stage confirmed in two continuous years), and (2) progression from Stage 1 to Stage 3 or 4 . The nephropathy endpoint was defined as development of overt nephropathy (spot urinary albumin excretion $>300 \mathrm{mg} / \mathrm{g}$ creatinine in two consecutive samples).

Macroangiopathy endpoints included the occurrence of definite CHD (angina pectoris or myocardial infarction) or stroke. Diagnosis of angina pectoris and myocardial infarction was according to criteria defined by the $\mathrm{WHO} /$ MONICA (Multinational Monitoring of Trends and Determinants in Cardiovascular Disease) project and diagnosis of stroke was according to guidelines defined by the Ministry of Health, Labour and Welfare of Japan [21]. Information regarding primary outcome and other clinical variables for each patient was collected through an annual report from each physician. Adjudication of endpoints was made by central committees comprising experts in each complication based on additional data such as CT or MRI of the brain or sequential changes in electrocardiograms.

Statistical analysis The sample size required to compare the net change in $\mathrm{HbA}_{1 \mathrm{c}}$ level (level at the third year point minus the baseline level) between the INT and CON groups is based on a consideration of power. It is assumed that the type I error $(\alpha)$ is 0.05 and the type II error $(\beta)$ is 0.1 ; therefore, in order to detect a difference of 0.2 in net change in $\mathrm{HbA}_{1 \mathrm{c}}$ level, with an $\mathrm{SD}$ of 1.0 among patients, a total sample size of 1,025 is required. In addition, if allowance is made for an up to $20 \%$ dropout rate, for $20 \%$ of the INT group being unable to complete the intervention and for $10 \%$ of the CON group to change treatment method within the follow-up period, the required sample size increases to 2,616 . Thus, in terms of the feasibility of the study, it was necessary to recruit more than 2,000 patients. All statistical analyses and data management were conducted at a central data centre. The Wilcoxon's rank sum test, Fisher's exact test and Mantel's test were used for comparison of numerical and categorical variables between groups.

The study endpoints were analysed as time-to-event variables, i.e. clinical data on patients who were lost during follow-up were used for the period for which they could be followed. Survival curves for the diabetic complications were estimated by the Kaplan-Meier method, and the logrank test was also conducted. Cox regression analysis was used to calculate the unadjusted and adjusted HRs and 95\% CIs for group and risk factors. In multivariate Cox analysis, all significant variables selected for the univariate 
analysis were used with the criterion of $p<0.1$. All values are presented as means \pm SD unless otherwise stated. All $p$ values are two-sided and the significance level is 0.05 . All statistical analyses were conducted using SAS packages ver. 9.1 (SAS Institute, Cary, NC, USA).

\section{Results}

Clinical variables and their changes Clinical characteristics of the patients at baseline and at the fourth and eighth year after the start of the study are shown in Table 1. There were no differences in most variables between the two groups except for the triacylglycerol level, which was slightly but significantly lower in the INT group at the eighth year. Proportions of patients using agents for hyperglycaemia, including insulin, hypertension and dyslipidaemia and anti-platelet agents did not differ significantly between groups. Frequency of clinic visits also did not differ between groups. Proportions of patients who satisfied all or each of the components of the therapeutic goals did not differ between groups at either the fourth or eighth year. Median follow-up time was 7.8 years.

Of the eligible patients, $73 \%$ were followed into the eighth year. The dropout rate, which was defined as the proportion of patients who were lost-to-follow up until the eighth year, in the INT group (24\%) was significantly lower than in the CON group (31\%). Significant differences in baseline characteristics between patients who completed (i.e. were followed until the end of the observation period) and did not complete (i.e. dropped out during the observation period) follow-up were only found in the proportion of patients on insulin ( $22 \%$ completed vs $18 \%$ did not complete, $p=0.03)$, in current smokers $(20 \%$ completed vs $7.5 \%$ did not complete, $p=0.01$ ) and amount of daily exercise $(590 \mathrm{~kJ} /$ day completed vs $351 \mathrm{~kJ} /$ day did not complete, $p<0.0001)$.

Effects of lifestyle modification There were no differences in energy or fat intake between groups in either the fifth or the eighth year of the study (Table 1). Physical activity as determined by the Baecke's Total Physical Activity Index [27] after 5 years of intervention was significantly higher in the INT group than in the CON group, with the difference in the total score being derived from the Sports Index (4.1 in the INT group vs 3.7 in the CON group, $p=0.028)$, but not Work or Leisure Indices. The proportion of current smokers in both groups decreased from $28 \%$ to $23 \%$, with no significance between groups.

Primary endpoint analysis During the study period, 345 retinopathy, 74 nephropathy, $115 \mathrm{CHD}$ and 90 stroke events occurred. Among all CHD events, $60 \%(n=69)$ were angina pectoris and $40 \%(n=46)$ were myocardial infarction, and among all stroke events, $83 \%(n=75)$ were brain infarction, $9 \%(n=8)$ were brain haemorrhage and $8 \%(n=7)$ were transient ischaemic attack. Kaplan-Meier curves for macroand microvascular endpoints are shown in Fig. 2, which demonstrates that the incidence of stroke in the INT group was significantly lower than that in the CON group.

Risk factors for stroke analysed by univariate and multivariate Cox proportional hazard models are shown in Table 2, and belonging to the INT group was associated with an approximately $40 \%$ significant risk reduction for stroke by both univariate and multivariate analyses when all significant variables determined by univariate analysis were included. Systolic BP and lipoprotein(a) were also significant factors that remained in multivariate analysis. Despite this, absolute values for BP and lipoprotein(a) did not differ significantly between groups. Even when myocardial infarction (including asymptomatic) or brain infarction was used as an endpoint instead of CHD or stroke, respectively, the above results were not changed (data not shown).

No group differences were found in the occurrence of CHD, development of retinopathy (35.7/1,000 patient-years in the CON group vs 39.0 in the INT group), progression of retinopathy $(6.5 / 1,000$ patient-years in the CON group vs 10.0 in the INT group) or development of nephropathy $(6.7 / 1,000$ patient-years in the CON group vs 6.7 in the INT group).

\section{Discussion}

Although lifestyle interventions in patients with type 2 diabetes have traditionally focused almost exclusively on weight loss, control of glycaemia and other major cardiovascular risk factors should also be considered simultaneously for the prevention of complications [28, 29]. Systematic reviews and meta-analyses have revealed clinically significant but considerably mild effects of lifestyle interventions on glycaemic control, that is about a $0.5 \%$ reduction in $\mathrm{HbA}_{1 \mathrm{c}}$ with some variations in $\mathrm{HbA}_{1 \mathrm{c}}$ levels depending on the study and its design $[8,11,22,23]$. In the Steno-2 study [16], the difference in mean $\mathrm{HbA}_{1 \mathrm{c}}$ levels between the conventional and intensive therapy groups after 7.8 years of follow-up was $1.1 \%$, with a marked reduction in many diabetic complications. This was accomplished through not only behavioural modification but also pharmacological therapy for control of glycaemia, $\mathrm{BP}$ and serum lipid levels. $\mathrm{HbA}_{1 \mathrm{c}}$ levels were not reported in previous studies that examined the effects of a lifestyle intervention on cardiovascular events in individuals with IGT $[13,14]$. The current study, together with our previous interim report [21], added the information that a lifestyle intervention produced significant but small and temporal 


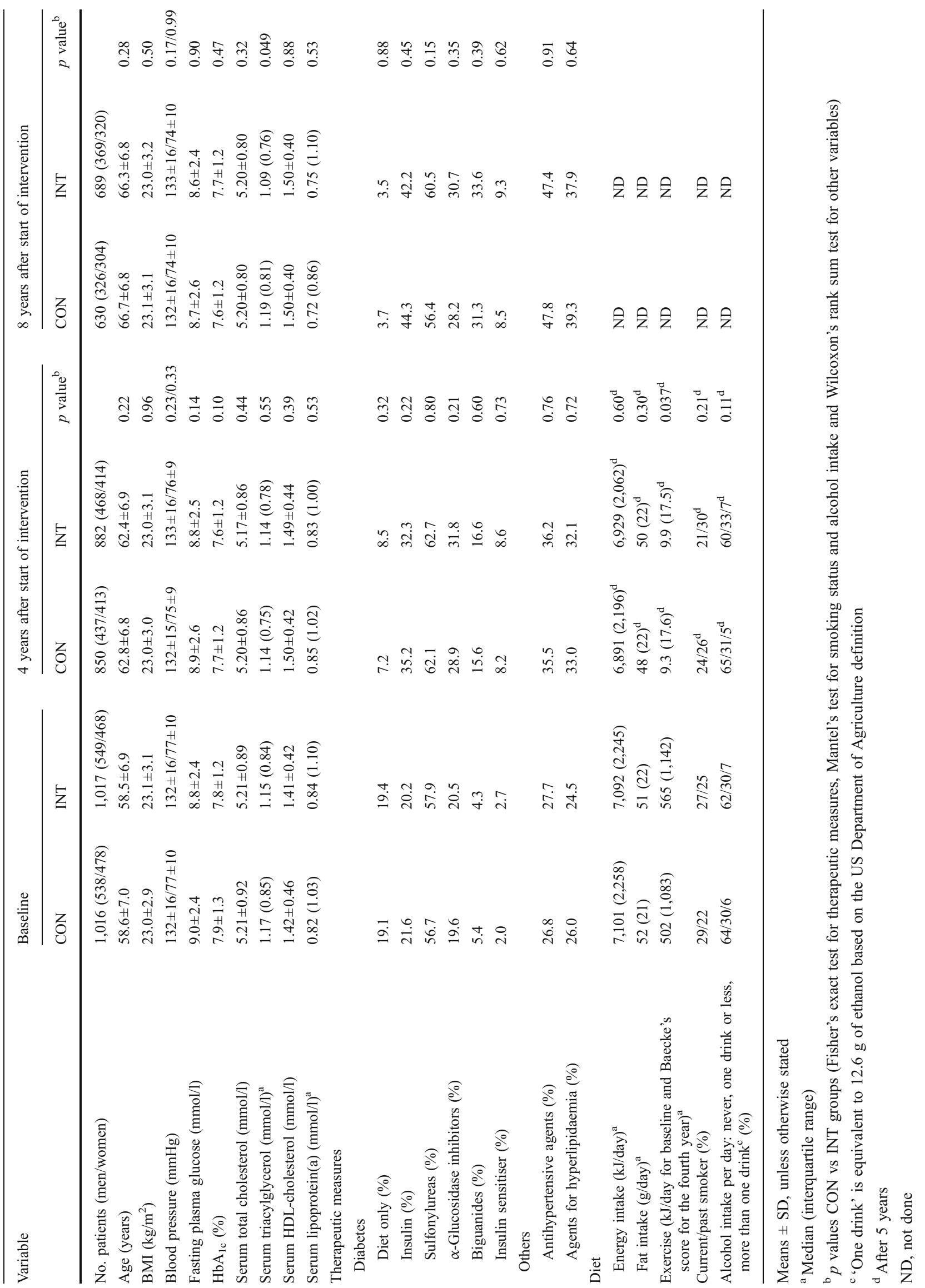



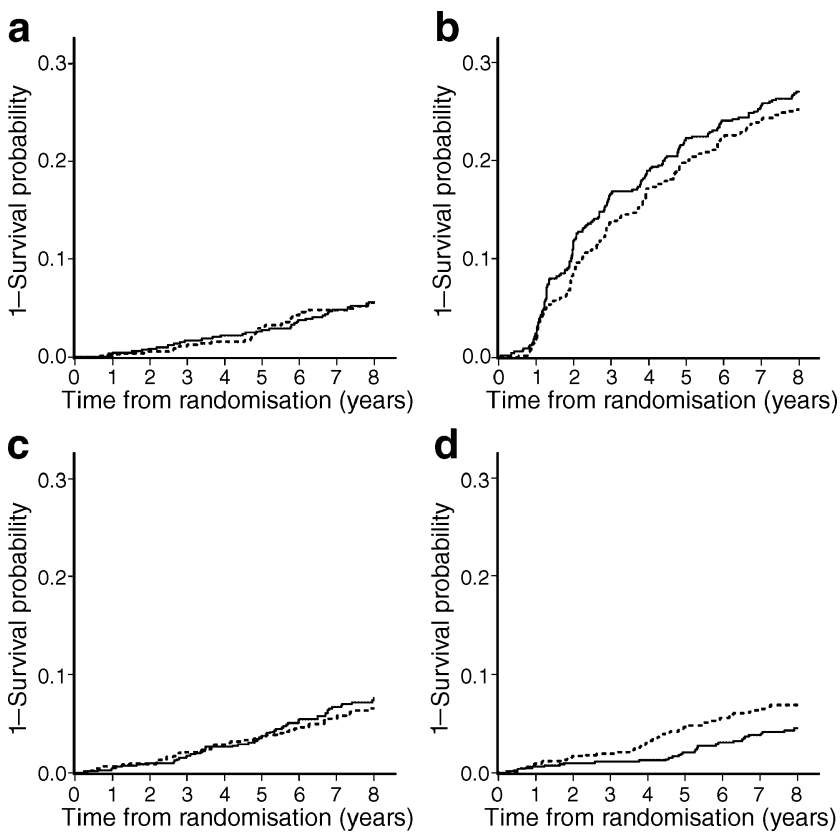

Fig. 2 Kaplan-Meier curves for each complication. a Nephropathy, $p=1.00$. b Retinopathy, $p=0.43$. c CHD, $p=0.40$. d Stroke, $p=0.02$. $p$ values by logrank test. Dotted curves, CON; solid curves, INT

improvement in glycaemic control and only minimal changes in other known risk factors for diabetic complications, indicating the difficulty in changing the lifestyle of patients with long-term diabetes.

One possible reason for these limited effects on lifestyle and risk factors was that the intensity of our lifestyle intervention, which consisted only of education, was considerably lower than that in other studies $[3,15]$. The rationale for this strategy was to determine if an intervention that is practicable to apply in the clinical 'real-world' setting with limited resources would be effective. Our results showed only very limited changes in actual lifestyle as well as major risk factors.

Another reason for the limited effects is that, in our study, even patients in the CON group received routine lifestyle education by diabetes specialists, which is an inevitable part of the usual care of persons with diabetes. Consequently, the effects of the lifestyle intervention was somewhat 'diluted'. The sequential increase in mean weight seen in the UK Prospective Diabetes Study (UKPDS) [30] was not observed in this study even in the CON group, indicating some effect of even routine lifestyle education. Also, perhaps if individualised goals with designated per cent changes for each patient in the INT group had been established instead of uniform goals for the entire group, results for the INT group might have been different. Further examination of results might have indicated which subgroups within the INT group would be most likely to benefit from such an intervention, which would be helpful in planning future interventions.
Nevertheless, we found a significant reduced risk for stroke in patients in the INT group compared with those in the CON group regardless of the lack of significant differences in most known cardiovascular risk factors. The mechanism of this apparent contradictory result is yet to be determined but it should be interpreted with care, especially since BP, which is a major risk factor for stroke, did not differ significantly between groups throughout the study period. Multifactorial or combined effects of lifestyle education/behaviours beyond individual factors [31] might have existed but can only be speculated upon. At the same time, the slight but significant differences in $\mathrm{HbA}_{1 \mathrm{c}}$ in the first 3 years, which was reported previously [21], but that disappeared thereafter, could enhance the effects since past interventions to lower $\mathrm{HbA}_{1 \mathrm{c}}$ reportedly have had a very long-term effect (i.e. 'metabolic memory' or 'legacy effect') $[32,33]$.

Other speculations for the apparent contradictory result include possible improvement in factors that were not determined in this study, such as postprandial glycaemia/ lipaemia, BP at home or psychological factors (stress,

Table 2 Risk factors for stroke analysed by Cox univariate and multivariate models

\begin{tabular}{|c|c|c|}
\hline Variable & $\operatorname{HR}(95 \% \mathrm{CI})$ & $p$ value \\
\hline \multicolumn{3}{|l|}{ Univariate analysis } \\
\hline Sex (women vs men) & $0.65(0.42-1.00)$ & 0.05 \\
\hline Age (per 10 years) & $1.53(1.11-2.13)$ & 0.01 \\
\hline Diabetes duration (per 10 years) & $0.95(0.70-1.28)$ & 0.72 \\
\hline $\mathrm{HbA}_{1 \mathrm{c}}($ per $1 \%)$ & $1.12(0.97-1.30)$ & 0.13 \\
\hline BMI (per $\left.1 \mathrm{~kg} / \mathrm{m}^{2}\right)$ & $1.05(0.98-1.12)$ & 0.18 \\
\hline Waist circumference (per $10 \mathrm{~cm}$ ) & $1.38(1.09-1.74)$ & 0.01 \\
\hline Systolic BP (per $10 \mathrm{mmHg}$ ) & $1.22(1.07-1.38)$ & $<0.01$ \\
\hline Diastolic BP (per $10 \mathrm{mmHg}$ ) & $1.18(0.96-1.45)$ & 0.12 \\
\hline LDL-cholesterol (per $1 \mathrm{mmol} / \mathrm{l}$ ) & $1.06(0.82-1.37)$ & 0.66 \\
\hline HDL-cholesterol (per $1 \mathrm{mmol} / \mathrm{l})$ & $0.62(0.37-1.06)$ & 0.08 \\
\hline Triacylglycerol (per 1 mmol/l) & $1.16(0.96-1.41)$ & 0.14 \\
\hline Lipoprotein(a) (per $1 \mu \mathrm{mol} / \mathrm{l})$ & $1.17(1.04-1.31)$ & 0.01 \\
\hline Current smoker (yes vs no) & $1.22(0.95-1.56)$ & 0.13 \\
\hline Alcohol intake (per $10 \mathrm{~g}$ ethanol) & $1.06(0.97-1.16)$ & 0.23 \\
\hline Exercise amount (per 418 kJ) & $1.01(0.93-1.09)$ & 0.80 \\
\hline INT group (vs CON group) & $0.61(0.39-0.93)$ & 0.02 \\
\hline \multicolumn{3}{|l|}{ Multivariate analysis } \\
\hline Sex (women vs men) & $0.68(0.42-1.11)$ & 0.12 \\
\hline Age (per 10 years) & $1.42(0.99-2.04)$ & 0.06 \\
\hline Systolic BP (per 10 mmHg) & $1.22(1.05-1.40)$ & 0.01 \\
\hline Lipoprotein(a) (per $1 \mu \mathrm{mol} / \mathrm{l})$ & $1.16(1.03-1.31)$ & 0.01 \\
\hline INT group (vs CON group) & $0.62(0.39-0.98)$ & 0.04 \\
\hline
\end{tabular}

All significant variables selected for the univariate analysis with the criterion of a $p<0.1$ were used in the multivariate analysis 
motivation or quality of life) [34], which could be ameliorated in the INT group rather than in the CON group. For example, Roumen et al. [35] recently reported that a lifestyle intervention successfully improved postprandial glucose levels in IGT patients. Changes in diet might also be effective, such as an increase in fruit intake, which is reportedly associated with reduced CVD mortality [36]. The reasons that only stroke, but not CHD or other complications, was found to be responsive to our intervention are speculated to include the following: (1) stroke is more frequent than CHD in Japan compared with other parts of the world, and (2) the independent risk factor for stroke was only systolic BP and lipoprotein(a), and so there would be room for other undetermined risk factors to work.

Telephone counselling in patients with chronic disease was shown to be associated with a $41 \%$ significant reduction in the risk of death [37]. However, attempts to use telephone calls in diabetes care have resulted in relatively mild [38, 39] or no additional [40] effects on control variables or improved quality of life [41] or patient satisfaction [39]. However, its effects on complication events have not been determined previously. Current results suggested that the telephone intervention could have contributed to a reduction in complication events. Further investigation is required to clarify whether telephone counselling alone is effective in improving the occurrence of complication events or death.

Lipoprotein(a), primarily a genetically determined risk factor for atherothrombogenesis, was found to be one significant predictor of stroke in our analysis. It has been reported as a predictor of deterioration of renal function [42], peripheral arterial disease [43], CVD [44] including CHD [45], and cardiovascular mortality [46] in patients with type 2 diabetes and a predictor of CVD [47] in patients with type 1 diabetes. It is of interest that the serum level of lipoprotein(a), which is known to be less affected by lifestyle or medication than other cardiovascular risk factors [48], was also a significant factor independent from lifestyle in our cohort.

The strengths of our study are that (1) it is the first intervention study mainly focused on the effects of lifestyle education on diabetic vascular complications, and (2) followup was done by diabetes specialists, ensuring that the quality of data was relatively high. Nevertheless, we acknowledge that the study had certain limitations. First, our participants were hospital-based patients with diabetes of a relatively long duration. Therefore, we cannot make inferences beyond a similar group. Second, only Asian diabetic patients were involved and they are different from other ethnic groups in terms of degree of obesity [49]. Third, we had a low follow-up rate, since the study was done mainly in large hospitals in urban areas where patients move quite frequently. However, it is less likely that this could be a cause of an inter-group difference in stroke incidence since significant differences in the incidence of stroke between groups could already be seen 4-5 years after the intervention began, when the follow-up rates of the two groups were not significantly different.

A therapeutic intervention mainly focused on lifestyle changes produced a significantly reduced risk of stroke in Japanese patients with type 2 diabetes independently of known classic risk factors. The detailed mechanisms for this effect should be investigated in the future.

Acknowledgements We thank the many diabetologists and patients at 59 participating institutes all over Japan. Thanks are extended to Y. Maruyama for her excellent secretarial assistance. This study was financially supported by the Ministry of Health, Labour and Welfare, Japan. The sponsor had no role in the design and conduct of the study.

Duality of interest The authors declare that there is no duality of interest associated with this manuscript.

Open Access This article is distributed under the terms of the Creative Commons Attribution Noncommercial License which permits any noncommercial use, distribution, and reproduction in any medium, provided the original author(s) and source are credited.

\section{References}

1. Pan XR, Li GW, Hu YH et al (1997) Effects of diet and exercise in preventing NIDDM in people with impaired glucose tolerance. The Da Qing IGT and Diabetes Study. Diabetes Care 20:537-544

2. Tuomilehto J, Lindstrom J, Eriksson JG et al (2001) Prevention of type 2 diabetes mellitus by changes in lifestyle among subjects with impaired glucose tolerance. $\mathrm{N}$ Engl J Med 344:1343-1350

3. Knowler WC, Barrett-Connor E, Fowler SE et al (2002) Reduction in the incidence of type 2 diabetes with lifestyle intervention or metformin. N Engl J Med 346:393-403

4. Kosaka K, Noda M, Kuzuya T (2005) Prevention of type 2 diabetes by lifestyle intervention: a Japanese trial in IGT males. Diabetes Res Clin Pract 67:152-162

5. Lindstrom J, Ilanne-Parikka P, Peltonen M et al (2006) Sustained reduction in the incidence of type 2 diabetes by lifestyle intervention: follow-up of the Finnish Diabetes Prevention Study. Lancet 368:1673-1679

6. Gillies CL, Abrams KR, Lambert PC et al (2007) Pharmacological and lifestyle interventions to prevent or delay type 2 diabetes in people with impaired glucose tolerance: systematic review and meta-analysis. BMJ 334:299-302

7. Conn VS, Hafdahl AR, Mehr DR, LeMaster JW, Brown SA, Nielsen PJ (2007) Metabolic effects of interventions to increase exercise in adults with type 2 diabetes. Diabetologia 50:913-921 
8. Davies MJ, Heller S, Skinner TC et al (2008) Effectiveness of the diabetes education and self management for ongoing and newly diagnosed (DESMOND) programme for people with newly diagnosed type 2 diabetes: cluster randomised controlled trial. BMJ 336:491-495

9. Christian JG, Bessesen DH, Byers TE, Christian KK, Goldstein MG, Bock BC (2008) Clinic-based support to help overweight patients with type 2 diabetes increase physical activity and lose weight. Arch Intern Med 168:141-146

10. Herder C, Peltonen M, Koenig W et al (2009) Anti-inflammatory effect of lifestyle changes in the Finnish Diabetes Prevention Study. Diabetologia 52:433-442

11. Norris SL, Lau J, Smith SJ, Schmid CH, Engelgau MM (2002) Self-management education for adults with type 2 diabetes: a meta-analysis of the effect on glycemic control. Diabetes Care 25:1159-1171

12. Loveman E, Cave C, Green C, Royle P, Dunn N, Waugh N (2003) The clinical and cost-effectiveness of patient education models for diabetes: a systematic review and economic evaluation. Health Technol Assess 7(iii): 1-190

13. Li G, Zhang P, Wang J et al (2008) The long-term effect of lifestyle interventions to prevent diabetes in the China Da Qing Diabetes Prevention Study: a 20-year follow-up study. Lancet 371:1783-1789

14. Uusitupa M, Peltonen M, Lindstrom J et al (2009) Ten-year mortality and cardiovascular morbidity in the Finnish Diabetes Prevention Study - secondary analysis of the randomized trial. PLoS ONE 4:e5656

15. Gaede P, Vedel P, Larsen N, Jensen GV, Parving HH, Pedersen O (2003) Multifactorial intervention and cardiovascular disease in patients with type 2 diabetes. N Engl J Med 348:383-393

16. Gaede P, Lund-Andersen H, Parving HH, Pedersen O (2008) Effect of a multifactorial intervention on mortality in type 2 diabetes. N Engl J Med 358:580-591

17. Sone H, Yoshimura Y, Ito H, Ohashi Y, Yamada N (2004) Energy intake and obesity in Japanese patients with type 2 diabetes. Lancet 363:248-249

18. Sone H, Mizuno S, Fujii $\mathrm{H}$ et al (2005) Is the diagnosis of metabolic syndrome useful for predicting cardiovascular disease in Asian diabetic patients? Analysis from the Japan Diabetes Complications Study. Diabetes Care 28:1463-1471

19. Sone H, Tanaka S, Ishibashi S et al (2006) The new worldwide definition of metabolic syndrome is not a better diagnostic predictor of cardiovascular disease in Japanese diabetic patients than the existing definitions: additional analysis from the Japan Diabetes Complications Study. Diabetes Care 29:145-147

20. Sone H, Tanaka S, Iimuro S et al (2009) Waist circumference as a cardiovascular and metabolic risk in Japanese patients with type 2 diabetes. Obesity 17:585-592

21. Sone H, Katagiri A, Ishibashi $S$ et al (2002) Effects of lifestyle modifications on patients with type 2 diabetes: the Japan Diabetes Complications Study (JDCS) study design, baseline analysis and three year-interim report. Horm Metab Res 34:509-515

22. Chodosh J, Morton SC, Mojica W et al (2005) Meta-analysis: chronic disease self-management programs for older adults. Ann Intern Med 143:427-438

23. Shojania KG, Ranji SR, McDonald KM et al (2006) Effects of quality improvement strategies for type 2 diabetes on glycemic control: a meta-regression analysis. JAMA 296:427-440

24. The Japan Diabetes Society (2002) Food exchange lists dietary guidance for persons with diabetes. Bunkodo, Tokyo

25. Shikoku University Nutrition Data Base. http://www2-eiyoashi koku-uacjp/ (in Japanese), accessed 1 Sep 2009
26. Ministry of Education C, Sports, Science and Technology, Japan (2004) Standard Tables of Food Composition in Japan. http:// wwwmextgojp/b_menu/shingi/gijyutu/gijyutu3/toushin/05031 802htm (in Japanese), accessed 1 Sep 2009

27. Baecke JA, Burema J, Frijters JE (1982) A short questionnaire for the measurement of habitual physical activity in epidemiological studies. Am J Clin Nutr 36:936-942

28. Klein S, Sheard NF, Pi-Sunyer X et al (2004) Weight management through lifestyle modification for the prevention and management of type 2 diabetes: rationale and strategies: a statement of the American Diabetes Association, the North American Association for the Study of Obesity, and the American Society for Clinical Nutrition. Diabetes Care 27: 2067-2073

29. Buse JB, Ginsberg HN, Bakris GL et al (2007) Primary prevention of cardiovascular diseases in people with diabetes mellitus: a scientific statement from the American Heart Association and the American Diabetes Association. Diabetes Care $30: 162-172$

30. The United Kingdom Prospective Diabetes Study Group (1998) Intensive blood-glucose control with sulphonylureas or insulin compared with conventional treatment and risk of complications in patients with type 2 diabetes (UKPDS 33). Lancet 352:837-853

31. Myint PK, Luben RN, Wareham NJ, Bingham SA, Khaw K-T (2009) Combined effect of health behaviours and risk of first ever stroke in 20, 040 men and women over 11 years' follow-up in Norfolk cohort of European Prospective Investigation of Cancer (EPIC Norfolk): prospective population study. BMJ. doi:10.1136/ bmj.b349

32. Nathan DM, Cleary PA, Backlund JY et al (2005) Intensive diabetes treatment and cardiovascular disease in patients with type 1 diabetes. N Engl J Med 353:2643-2653

33. Holman RR, Paul SK, Bethel MA, Matthews DR, Neil HA (2008) 10 -year follow-up of intensive glucose control in type 2 diabetes. N Engl J Med 359:1577-1589

34. Araki A, Murotani Y, Kamimiya F, Ito H (2004) Low well-being is an independent predictor for stroke in elderly patients with diabetes mellitus. J Am Geriatr Soc 52:205-210

35. Roumen C, Corpeleijn E, Feskens EJ, Mensink M, Saris WH, Blaak EE (2008) Impact of 3-year lifestyle intervention on postprandial glucose metabolism: the SLIM study. Diabet Med 25:597-605

36. Nothlings U, Schulze MB, Weikert $C$ et al (2008) Intake of vegetables, legumes, and fruit, and risk for all-cause, cardiovascular, and cancer mortality in a European diabetic population. J Nutr 138:775-781

37. Wu JY, Leung WY, Chang $\mathrm{S}$ et al (2006) Effectiveness of telephone counselling by a pharmacist in reducing mortality in patients receiving polypharmacy: randomised controlled trial. BMJ 333:522-525

38. Piette JD, Weinberger M, McPhee SJ, Mah CA, Kraemer FB, Crapo LM (2000) Do automated calls with nurse follow-up improve self-care and glycemic control among vulnerable patients with diabetes? Am J Med 108:20-27

39. Piette JD, Weinberger M, Kraemer FB, McPhee SJ (2001) Impact of automated calls with nurse follow-up on diabetes treatment outcomes in a Department of Veterans Affairs Health Care System: a randomized controlled trial. Diabetes Care 24:202-208

40. Lorig K, Ritter PL, Villa F, Piette JD (2008) Spanish diabetes selfmanagement with and without automated telephone reinforcement: two randomized trials. Diabetes Care 31:408-414

41. Maljanian R, Grey N, Staff I, Conroy L (2005) Intensive telephone follow-up to a hospital-based disease management model for patients with diabetes mellitus. Dis Manag 8:15-25 
42. Song KH, Ko SH, Kim HW et al (2005) Prospective study of lipoprotein(a) as a risk factor for deteriorating renal function in type 2 diabetic patients with overt proteinuria. Diabetes Care 28:1718-1723

43. Tseng CH (2004) Lipoprotein(a) is an independent risk factor for peripheral arterial disease in Chinese type 2 diabetic patients in Taiwan. Diabetes Care 27:517-521

44. Hiraga T, Kobayashi T, Okubo M et al (1995) Prospective study of lipoprotein(a) as a risk factor for atherosclerotic cardiovascular disease in patients with diabetes. Diabetes Care $18: 241-244$

45. Murase T, Okubo M, Amemiya-Kudo M, Ebara T, Mori Y (2008) Impact of elevated serum lipoprotein (a) concentrations on the risk of coronary heart disease in patients with type 2 diabetes mellitus. Metabolism 57:791-795

46. Hernandez C, Francisco G, Chacon P, Simo R (2005) Lipoprotein(a) as a risk factor for cardiovascular mortality in type 2 diabetic patients: a 10-year follow-up study. Diabetes Care 28:931-933

47. Kollerits B, Auinger M, Reisig V et al (2006) Lipoprotein(a) as a predictor of cardiovascular disease in a prospectively followed cohort of patients with type 1 diabetes. Diabetes Care 29:1661-1663

48. Scanu AM, Lawn RM, Berg K (1991) Lipoprotein(a) and atherosclerosis. Ann Intern Med 115:209-218

49. Sone H, Ito H, Ohashi Y, Akanuma Y, Yamada N (2003) Obesity and type 2 diabetes in Japanese patients. Lancet 361:85 